\title{
POTENTIALS PRODUCING MAXIMALLY SHARP RESONANCES
}

\author{
BY \\ EVANS M. HARRELL II ${ }^{1}$ AND ROMAN SVIRSKY ${ }^{2}$
}

\begin{abstract}
We consider quantum-mechanical potentials consisting of a fixed background plus an additional piece constrained only by having finite height and being supported in a given finite region in dimension $d \leqslant 3$. We characterize the potentials in this class that produce the sharpest resonances. In the one-dimensional or spherically symmetric specialization, a quite detailed description is possible. The maximally sharp resonances that we find are, roughly speaking, caused by barrier confinement of a metastable state, although in some situations they call for interactions in the interior of the confining barrier as well.
\end{abstract}

I. Introduction. One of the standard topics of quantum mechanics is the tunneling effect. A large potential barrier blocks a particle imperfectly, and the effect of the penetration can show up in scattering as a sharp resonance. In the time-independent analysis of the Schrödinger equation, resonances make their appearance in the guise of nonreal eigenvalues defined with an outgoing-wave condition or complex scaling. Up to physical constants, $\varepsilon$, which will denote (minus) the imaginary part of this eigenvalue, measures the width of the resonance in units of energy, and a sharp resonance is one with small $\varepsilon$. The real part, $E$, roughly locates the physical energy at which the resonance is observed. The quantity $\varepsilon$ may also be inversely proportional to the lifetime of a metastable state, according to the indeterminacy principle. We shall consider relatively compact potentials $V$ supported in finite regions in one or three dimensions, which are exterior-dilatation analytic in the sense described by Simon and by Graffi and Yajima $[14,6]$. They also seem to fall within the scope of other recent generalizations of the complex scaling method $[3,4,10,13]$, although we have not yet seen the definitive versions of all of these generalizations. The simplest model of an alpha-emitting nucleus, being a spherical square-well, fits this description, and its sharp resonances are associated with the metastable states caused physically by confinement of particles within the nucleus by a potential barrier at its periphery. It is not obvious, however, that other mechanisms might not also exist for causing resonances. For instance, could some very complicated potential, such as arises in studies of random media, cause as sharp a scattering resonance as a confining barrier? We will find below that the answer is in essence no.

Received by the editors April 10, 1985. This paper was partly presented at the Colloque sur les Méthodes Semi-Classique en Méchanique Quantique, September 10-15, 1984, at C.I.R.M., Luminy, France.

1980 Mathematics Subject Classification. Primary 81C12; Secondary 34B25.

${ }^{1}$ Partially supported by USNSF Grant MCS 8300551 and an Alfred P. Sloan Fellowship.

${ }^{2}$ This work is partially based on the second author's Johns Hopkins Ph.D. dissertation, June, 1985. 
This paper uses and extends ideas in two earlier works by Harrell [7, 8]. In [7] Harrell studied the one-dimensional Schrödinger equation

$$
-d^{2} \psi / d x^{2}+\left(V(x)-k^{2}\right) \psi=0, \quad k^{2}=E-i \varepsilon,
$$

with outgoing boundary conditions at 0 and an arbitrary other fixed point $L$. Positive lower bounds were derived for $\varepsilon$ depending only on the support $\subset[0, L]$ and magnitude of $V$ and on the real part of the resonance eigenvalue $E$, which therefore apply to random or otherwise imperfectly known potentials. That article relied on comparison techniques to generate inequalities, but an alternative approach, which we follow here, is to attempt actually to find the most highly resonant possible potential within some category. This could then be analyzed, if necessary numerically, to furnish optimal bounds on $\varepsilon$. Harrell's other paper [8] investigated the problem of determining the potential that optimizes a different spectral property, namely the ground-state eigenvalue of an $n$-dimensional Schrödinger operator, and further progress on related problems was made recently in [2]. This provides both a method and a reason for hoping for success in the resonance problem, which is, however, in many ways less tractable, especially because it is not selfadjoint.

In this paper we study equation (1.1) and its higher-dimensional analogue,

$$
(-\Delta+V(x)) \psi=k^{2} \psi .
$$

In the one-dimensional case we shall pose slightly different boundary conditions from those of [7], viz.,

$$
\psi(0)=0 \quad \text { and } \quad \psi(L)=1, \quad \psi^{\prime}(L)=i k,
$$

i.e., Dirichlet conditions at 0 and the traditional outgoing conditions at $L$. The lower bound derived in [7], which assumed outgoing conditions at both endpoints, carries over immediately with only minor changes. Boundary conditions (1.3) are appropriate if one thinks of the one-dimensional problem as coming from separation of variables in a spherically symmetric three-dimensional problem, and would describe $S$-wave resonances; it will thus be referred to as the totally spherically symmetric case. Resonances for subspaces of nonzero angular momentum would correspond to an outgoing condition of the form

$$
\psi^{\prime}(L) / \psi(L) \rightarrow i k \quad \text { as } L \rightarrow \infty
$$

and will be discussed further in [15].

Since the boundary conditions (1.3) depend on the eigenvalue parameter $k^{2}$, it looks at first as if (1.1) and (1.3) do not constitute an operator eigenvalue problem, but in fact it is easy to show that these equations are equivalent to the eigenvalue problem for the one-dimensional, exteriorly dilated version of the operator $-\Delta+V$, since any eigensolution reduces to a plane wave $C \exp (i k x)$ in the region exterior to the potential but interior to the sphere where exterior dilatation sets in. To sum up, for our purposes:

Definition. A resonance is a triple $\left\langle k^{2}, V(x), \psi(x)\right\rangle$ related by (1.2) and the auxiliary conditions mentioned above, with $\operatorname{Re} k^{2} \geqslant 0$ and $\operatorname{Im} k^{2}<0$. We shall frequently refer to $k^{2}$ for short as the resonance, and will call $\psi$ (either as a local solution or as an exteriorly dilated solution) the resonance wave-function. 
We shall address the following question: Is there a distinguished potential $V_{\#}$ within a class such as $\{V: 0 \leqslant V \leqslant M, \operatorname{supp} V \subset x:|x| \leqslant L\}$ that minimizes $\varepsilon$ in (1.1) or (1.2), and, if so, what is this maximally resonant $V_{\#}$ ? We shall also address the question of existence and characterization of potentials that are maximally resonant within a given energy range, and allow a fixed background potential. An analysis of similar questions with other natural classes $S$ over which the potential can vary will appear in [15].

A compactness argument will answer the first question in the affirmative, and to characterize $V_{\#}$ we shall begin by analyzing the effect of small perturbations of it, following an idea of [8]. This will give a certain amount of information about $V_{\#}$; in particular, it will reveal that for the above-mentioned class, $V_{\#}$ can only equal 0 or $M$. To get more detailed information on the nature of its support, however, we have to restrict ourselves to the spherically symmetric case and rely on techniques of ordinary differential equations.

II. Preliminaries. The first order of business is to establish the existence of sharp resonances for suitable Schrödinger operators. We shall work in the spaces $\mathbf{R}^{+}, \mathbf{R}^{2}$, or $\mathbf{R}^{3}$, and always suppose that the potential $V$ is supported within the ball of radius $L$ centered at the origin. In one dimension this statement will be interpreted as meaning that $\operatorname{supp}(V) \subset[0, L]$. The exterior-wave condition can be incorporated into the eigenvalue problem

$$
-\Delta \psi+V \psi=k^{2} \psi
$$

most conveniently when the latter is written as an integral equation,

$$
\psi=-\int_{|y| \leqslant L} G(x, y ; k) V(y) \psi(y) d y,
$$

where we continue onto the second sheet, i.e., with $E=\operatorname{Re}\left(k^{2}\right)>0$ and $\varepsilon=$ $-\operatorname{Im}\left(k^{2}\right)>0$

$$
G(x, y ; k)= \begin{cases}\exp \left(i k x_{>}\right) \sin \left(k x_{<}\right) / k, & d=1, \\ i H_{0}^{(1)}(k|x-y|) / 4, & d=2, \\ \exp (i k|x-y|) / 4 \pi|x-y|, & d=3\end{cases}
$$

(here $H$ denotes a Hankel function [16]). We observe that any solution of (2.2) belongs to $W^{2}(\Omega)$ for any bounded domain $\Omega$ and solves (2.1).

What complex scaling provides for us is a consistent interpretation, in the language of operators on $L^{2}$, of this traditional method of defining a resonance. The only facts needed about the exterior scaling formalism are (i) that the associated resonance wave-functions satisfy the Schrödinger equation locally but are modified outside some finite region so as to become square-integrable; and (ii) if $J$ is the antilinear operator of complex conjugation, $J f=f$, then the adjoint of a complexscaled Hamiltonian operator $H_{d}$ is simply

$$
H_{d}^{*}=J H_{d} J .
$$

This prefatory remark should make it clear that our analysis is not strictly tied to 
the exterior-scaling formalism, but would apply without change to the other alternative complex-scaling techniques that have sprung up recently $[3, \mathbf{4}, \mathbf{1 0}, \mathbf{1 3}]$. Since we make only fairly minor use of complex scaling (to justify perturbation theory in Proposition III.1), the detailed discussion of the relationship between it and the integral equation is deferred to [15].

It will be helpful to know that there are very sharp resonances for sufficiently large support or potential height, i.e., that $\varepsilon$ is exponentially small as a function of these quantities. Suppose that $V$ is supported in the ball of radius $L$ and that $\operatorname{supp}|V| \leqslant M$. There is a scaling relationship between $L$ and $M$ showing that the problem is largely characterized by the combination $L \sqrt{M}$; if $x$ is replaced by $x^{\prime}=a x$, one finds that the length $L$ becomes $a L$, while the potential added to $-\Delta^{\prime}$ becomes $V\left(x^{\prime} / a\right) / a^{2}$. (The corresponding eigenvalue will also be affected, becoming $k^{2} / a^{2}$.) For convenience, in one dimension we may therefore show the existence of sharp resonances by setting $V=M \chi_{[1.2]}$, a standard textbook variety square-well. It is straightforward to find that the width of the principle resonance is exponentially small, i.e., $\exp (-2 \sqrt{M}$ ) as $M \rightarrow \infty$. (A rigorous discussion of this sort of limit, complete with detailed perturbation theory for large barriers of general shape, can be found in [1].) For the square barrier $M \chi_{[1, L]}$, there is a resonance whose width is asymptotic to $A \exp (-2 L \sqrt{M})$.

Similar analysis of spherically symmetric square-barrier potentials in dimensions 2 and 3 shows that in all cases there are universal positive constants $A$ and $B$, such that a potential $V, 0 \leqslant V \leqslant M$, supported in a ball of radius $L$, can always be found with a resonance width satisfying

$$
\varepsilon<A \exp (-B L \sqrt{M}) \text {. }
$$

If necessary, estimates of $A$ and $B$ could be derived without much difficulty. In the totally spherically symmetric case, for example, for any positive $A$ and any $B<2$, there is a resonance for which (2.5) will hold for $L$ or $M$ sufficiently large.

Fix a function $W$ supported within the ball of radius $L$ and a compact subset $\Omega$ of that ball. The function $W$ will play the role of a background potential and will be assumed relatively compact with respect to $-\Delta$. (This will be the case if $W \in L^{2}$, for example.) Let

$$
S=\{V: \operatorname{supp}(V) \subset \Omega \text { and } 0 \leqslant V(x)-W(x) \leqslant M \text { a.e. }\},
$$

let $\varepsilon(V)$ denote any particular resonance width associated with $V$, and let $E(V)$ be the real part of the corresponding eigenvalue $k^{2}(V)=E(V)-i \varepsilon(V)$ of $-\Delta+V$.

THEOREM II.1. Let $\varepsilon_{\#}=\inf \{\varepsilon(V): V \in S(C, D)\}$, where $S(C, D)$ is the subset of $S$ such that $0 \leqslant C \leqslant E(V) \leqslant D<\infty$. We assume $C$ and $D$ are chosen so that $\varepsilon_{\#}$ is defined (i.e., that there is a $V$ with a resonance eigenvalue in this energy interval). Then

(i) There exists a $V_{\#} \in S$ such that $\varepsilon_{\#}=\varepsilon\left(V_{\#}\right)$ and $C \leqslant E(V) \leqslant D$.

(ii) If either $W \geqslant 0$ a.e. or $C>0$, then $\varepsilon_{\#}>0$.

REMARK. There is no guarantee of uniqueness for the maximally resonant potential, and we expect that there are situations where it is not unique. For instance, suppose that $\Omega$ consists of two widely separated disjoint symmetric pieces. There is 
no physical reason to think that a resonance that would be sharp if only one piece were allowed would necessarily be enhanced if the second piece were equipped with a symmetric bit of potential. On the other hand, we conjecture that the typical situation is uniqueness.

Proof. Let $\Omega_{1}$ be an arbitrary finite closed ball containing $\Omega$. Let $V_{n}$ be a minimizing sequence for $\varepsilon$, i.e., $\varepsilon\left(V_{n}\right) \rightarrow \varepsilon_{\#}$. Let $k_{n}^{2}$ and $\psi_{n}$ be the associated eigenvalue and eigenfunction. Without loss of generality, since $[C, D]$ is a compact interval, we can pass to a subsequence so that $k_{n}^{2}$ converges. If $\psi_{n}$ is normalized in $L^{2}\left(\Omega_{1}\right)$, then (2.1) shows that $\psi_{n}$ lies in a bounded set in $W^{2}\left(\Omega_{1}\right)$. By Rellich's theorem this is compactly embedded in $C\left(\Omega_{1}\right)$, so by passing to another subsequence if necessary, it may be assumed that $\psi_{n}$ converges uniformly on $\Omega_{1}$. With still another subsequence, we may suppose by the Alaoğlu theorem that $V_{n}$ converges weakly in $L^{2}\left(\Omega_{1}\right)$, say to $V_{\#}$. The limit clearly remains in the set $S$ (integrate $V_{n}$ by the charactistic function of the set on which putatively $V_{\#}-W<0$ or $V_{\#}-W>$ $M)$.

Now note that $V_{n} \psi_{n}$ tends weakly to $V_{\#} \psi_{\#}$. For fixed $x \in \Omega_{1}$, the Green function tends to $G\left(x, y ; k_{\#}\right)$ in $L^{2}\left(\Omega_{1}, d y\right)$, so it follows that the right side of

$$
\psi_{n}(x)=-\int_{\Omega_{1}} G\left(x, y ; k_{n}\right) V_{n}(y) \psi_{n}(y) d y
$$

from (2.2) converges pointwise to

$$
-\int G\left(x, y ; k_{\#}\right) V_{\#}(y) \psi_{\#}(y) d y .
$$

The left side converges uniformly on $\Omega_{1}$ to $\psi_{\#}$, so

$$
\psi_{\#}(x)=-\int_{\Omega_{1}} G\left(x, y, k_{\#}\right) V_{\#}(y) \psi_{\#}(y) d y
$$

on $\Omega_{1}$.

If the minimal value of $\varepsilon$ were 0 , then the corresponding eigenvalue $k_{\#}^{2}$ would either be 0 or a positive embedded real eigenvalue of the selfadjoint realization of the problem (1.2) by the usual argument of dilatation analyticity (see [12, §XIII.13], which extends in a straightforward way to exterior scaling). Embedded positive eigenvalues, however, are impossible for bounded, compactly supported potentials (see [12, §XIII.13 or 5$]$ ).

It remains to show that if $W \geqslant 0$, there can be no eigenvalue or resonance with $k^{2}=0$. We consider the three-dimensional case only. Suppose the contrary. Then we would have

$$
\psi_{\#}=-(1 / 4 \pi|x|) * V_{\#} \psi_{\#},
$$

and because $V_{\#}$ is compactly supported it would follow that this produces a solution of the Schrödinger equation (without exterior scaling) tending to 0 at $\infty$. Since (see $[12$, vol. II, p. 183]) in general

$$
\Delta|u| \geqslant \operatorname{Re}((\bar{u} /|u|) \Delta u)
$$

it follows in this case that

$$
\Delta\left|\psi_{\#}\right| \geqslant V_{\#}\left|\psi_{\#}\right| \geqslant 0
$$


Therefore $\left|\psi_{\#}\right|$ satisfies the subharmonic maximum principle, and so cannot vanish at $\infty$ without being identically zero.

Theorem II.1 leaves open the possibility that the maximally resonant potential in any energy interval $[C, D]$ always has real part $C$ or $D$. This would mean either that the sharpest resonances always occurred at zero energy (i.e., $E\left(V_{\#}\right)=0$ ) or that resonances could always be made sharper by increasing the energy indefinitely. Both possibilities can be ruled out in cases of interest.

Proposition II.2. Consider the totally spherically symmetric case, and suppose that $W(r) \geqslant 0$ a.e. Then:

(a) Any zero-energy resonance has a width satisfying $\varepsilon>9 / 2 L^{2}$.

(b) Moreover, every resonance with

$$
\varepsilon<\max \left(\sqrt{E} / L, 1 / 2 L^{2}\right)
$$

satisfies

$$
E>\pi^{2} / 4 L^{2}
$$

Remarks. We expect that similar facts can also be proved without spherical symmetry, and even in this case the numbers could be improved somewhat. For sufficiently large $\sqrt{M} L$, this means that zero-energy or low-energy resonances, if they exist, are not maximal when the potential is forced to be nonnegative. If the potential can be negative, then zero-energy bound states and sharp resonances are entirely possible.

Proof. (a) Any zero-energy resonance has a subharmonic resonance wave-function, since from (2.7), as in (2.8)

$$
\Delta|\psi| \geqslant \operatorname{Re}(V-i \varepsilon)|\psi|=V|\psi| \geqslant 0 .
$$

It thus attains its maximum modulus on the boundary. With the normalization (1.3) the boundary values at $r=0$ and $r=L$ are 0 and $L$, so the subharmonic function $|\psi|$ must even be $\leqslant r / L$. A formula of [7] relates the resonance width to the corresponding resonance wave-function on $[0, L]$ and $K=\operatorname{Re} k$, viz.,

$$
\varepsilon=K / \int_{0}^{L}|\psi|^{2} d r
$$

(the extra term in [7] drops out because of the Dirichlet condition at 0 ). Since $\operatorname{Re} k^{2}=0, K=\sqrt{\varepsilon / 2}$, so (2.11) becomes

$$
\varepsilon=1 / 2\left[\int_{0}^{L}|\psi|^{2} d r\right]^{2}>1 / 2\left[\int_{0}^{L} r^{2} L^{-2} d r\right]^{2}=9 / 2 L^{2} .
$$

(b) Let $I=\int_{0}^{L}|\psi(r)|^{2} d r$. From (2.11) and $\varepsilon=2 K \sqrt{K^{2}-E}$, where $K=\operatorname{Re} k$, it follows that

$$
\varepsilon=K^{2} / I^{2} 2 K \sqrt{K^{2}-E}>1 / 2 I^{2} .
$$

Since $\sqrt{E}<K$, (2.9) implies that either $K / L>K / I$ or $1 / 2 L^{2}>1 / 2 I^{2}$, so in either case $I>L$. Since $|\psi|=0$ at 0 and 1 at $L$, it must attain values greater than 1 and thus have a maximum at some point $R \in(0, L)$. From (2.7), $|\psi|^{\prime \prime} \geqslant-E|\psi|$. 
Let $f=|\psi(R)| \cos (\sqrt{E}(r-R))$, so $f^{\prime \prime}=-E f$, while $f(R)=|\psi(R)|$ and $f^{\prime}(R)$ $=|\psi(R)|^{\prime}$. The Sturm comparison argument now leads to the conclusion that any zero of $|\psi(r)|$ for $r<R$ must lie to the left of the nearest zero of $f(r)$ (see [9, p. $334]$ ). Since $\psi(0)=0$, this means that $\sqrt{E} R \geqslant \pi / 2$, from which (2.10) follows.

As for the other regime of high energies, it is known that generally resonance eigenvalues are excluded from a sector in the complex plane of the form $\{0>$ $\left.\arg \left(k^{2}-\alpha\right)>-\beta\right\}$ for some positive $\alpha$ and $\beta$. The estimates used by Cycon [4], for example, to prove this fact hold uniformly for all $V \in S$. (Although Cycon uses a distorted scaling rather than exterior scaling, the distinction is unimportant in our context.)

COROLLARY II.3. In the totally spherically symmetric case, if $W \geqslant 0$ a.e. and $M$ or $L$ is sufficiently large, then there exists a potential $V_{\#}$ that is maximally resonant for the entire range of energies $E(V) \geqslant 0$, and $E\left(V_{\#}\right)>\pi^{2} / 4 L^{2}$.

Definition. The resonance $\left\langle k_{\#}^{2}, V_{\#}, \psi_{\#}\right\rangle$ with the potential asserted by II.3 to exist will be called the sharpest resonance of all.

III. Characterization of maximally resonant potentials. If a potential is maximally resonant on a set $S(C, D)$, then we term the corresponding resonance maximally sharp, or simply maximal. Thus a resonance is maximal when $\varepsilon$ is minimal. It was shown in §II that maximally resonant potentials exist under some physically important circumstances. Suppose now that $V_{\#}$ is a maximally resonant potential. It will be characterized by a variational analysis, which would equally well characterize minimally resonant potentials or other critical points of the functionals $\varepsilon(V)$. There is no apparent physical significance to other critical points, however. Since the sets $S$ and $S(C, D)$ which we consider here ensure that $V_{\#}$ is relatively compact with respect to the exteriorly complex dilated version of $-\Delta$, the resonances associated with $V_{\#}$ are all finitely degenerate and can accumulate only at $\infty$ or 0 . They will always be nondegenerate in the totally spherically symmetric case, and for simplicity we shall restrict ourselves to the problem of characterizing those maximally resonant potentials that have nondegenerate resonance eigenvalues. The functional configuration of $V_{\#}$ can be probed with small perturbations by appropriate functions. Since this variational analysis is purely local, a convenient definition reads as follows:

Definition. The potential $V_{\#}$ is locally maximally resonant for the set $S$ (or $S(C, D))$ if it has a resonance eigenvalue $k^{2}\left(V_{\#}\right)$ such that for sufficiently small $\delta$,

$$
\varepsilon\left(V_{\#}\right)=\min \left\{\varepsilon(V): V \in S, \sup \left|V-V_{\#}\right|<\delta,\left|k^{2}(V)-k^{2}\left(V_{\#}\right)\right|<\delta\right\} .
$$

The standard methods of perturbation theory allow one to write down a formula for the first-order change in $k^{2}$ when $V_{\#}$ is slightly perturbed, which will be a valuable tool:

Proposition III.1. Let $P(x)$ be a bounded, real function supported in $\Omega$. If $k^{2}$ is a discrete, nondegenerate resonance eigenvalue of $-\Delta+V, V \in S$, and $\psi_{d}$ is the associated eigenfunction $\in L^{2}$ in the framework of exterior dilatation, then

$$
d k^{2}\left(V_{d}+\kappa P\right) / d \kappa=\int P \psi_{d}^{2} / \int \psi_{d}^{2} .
$$


REMARK. With the usual complication of preliminary diagonalization, this formula remains valid for a finitely degenerate eigenvalue.

Proof. We write $k^{2}(V+\kappa P)$ for short as $k^{2}(\kappa)$ and let $H_{d}$ denote the exteriorly scaled version of $-\Delta+V$ for some fixed scaling parameter. From

$$
\left(k^{2}(\kappa)-k^{2}(0)\right)\left(J \psi_{d}, \psi_{d}\right)=\left(J \psi_{d},\left(H_{d}+\kappa P-k^{2}(0)\right) \psi_{d}\right),
$$

and the differentiability of $k^{2}$ and the eigenfunction guaranteed by perturbation theory [11, Chapter VII],

$$
\begin{aligned}
k^{2 \prime}(0)\left(J \psi_{d}, \psi_{d}\right) & =\left(d J \psi_{d} / d \kappa, 0\right)+\left(J \psi_{d}, P \psi_{d}\right)+\left(J \psi_{d},\left(H_{d}-k^{2}(0)\right) d \psi_{d} / d \kappa\right) \\
& =\left(J \psi_{d}, P \psi_{d}\right)+\left(\left(H_{d}^{*}-\overline{k^{2}(0)}\right) J \psi_{d}, d \psi_{d} / d \kappa\right)
\end{aligned}
$$

SO

$$
\left(d k^{2}(\kappa) / d \kappa\right) \int \psi_{d}^{2}=\int P \psi_{d}^{2}
$$

But note that $\int \psi_{d}^{2} \neq 0$, as otherwise the right side would be zero for all the functions $P$, implying that $\psi_{d}^{2}=0$ throughout $\Omega$, which is impossible because of the unique continuation property. Therefore we may divide through by the integral, obtaining (3.1).

THEOREM III.2. Let $V_{\#}$ be a maximally resonant potential in the set $S$. Then

$$
V_{\#}-W=M \chi_{Y} \text { a.e. }
$$

except possibly for $x$ on the nodal surface of the corresponding resonance wave function $\left\{x: \psi_{\#}(x)=0\right\}$.

REMARK. This fact is at first somewhat misleading about the nature of highly resonant potentials, since alternative types of maximally resonant potentials, such as are obtained when $V$ varies over a set with $L^{p}$ conditions rather than boundedness, turn out to be smooth functions characterized by nonlinear differential equations rather than (3.2) [15]. In other words, the discontinuity and two-valuedness of the maximally resonant potential are to some extent artifacts of the particular framework we have erected here. One great advantage that (3.2) brings is numerical feasibility. If a numerical estimate of the minimal resonance width is desired for a potential supported in a given region, the search procedure over this restricted set of potentials is easy to implement. In the spherically symmetric case the maximizers can be further characterized by analytic methods (see $§ I V$ ).

The nodal surface is necessarily of measure 0 if $V_{\#}$ is spherically symmetric, and is in any case a nowhere dense set, because of the unique continuation property.

Proof. Suppose not, and let $F_{n}=\left\{x: 0<1 / n<V_{\#}(x)-W(x)<M-1 / n\right\}$ for an arbitrary integer $n$. For uncluttered notation we call the associated wave-function simply $\psi$. Recall that $\psi$ and its exteriorly dilated version $\psi_{d}$ coincide within the undilated region. For almost every $z \in F_{n}$, we can find a sequence of subsets $G_{i} \subset F_{n}$ so that $\mu\left(G_{i}\right) \rightarrow 0$, and

$$
\psi^{2}=\lim _{i \rightarrow \infty} \int_{G_{i}} \psi^{2} d y / \mu\left(G_{i}\right)
$$


Now let $P_{i}(z)$ be the characteristic function of $G_{i}$; for $\kappa<1 / n, 0<V_{\#}-W+$ $\kappa P_{i}(x)<M$, so $\kappa P_{i}(x)$ is an admissible perturbation for sufficiently small positive or negative $\kappa$. If $V_{\#}$ is maximally resonant, then $\operatorname{Im} d k^{2}\left(V_{\#}+\kappa P_{i}\right) / \mathrm{d} \kappa=0$. From (3.1) and (3.3) this means that $\psi^{2} / \int \psi_{d}^{2} \equiv \alpha \psi^{2}$ is real for a.e. such $z$ (the denominator must contain the dilated wave-function in order to be finite). Since $n$ is arbitrary, we conclude that $\alpha \psi^{2}$ is purely real for a.e. $z \in F \equiv \bigcup F_{n}$.

Consider a point $z$ where, for instance, $\sqrt{\alpha} \psi(z)>0$. We claim that for a.e. such $z \in F$ we can find subsequences $\left\{z_{n}\right\}$ of points of $F$ converging to $z$ from $d$ linearly independent directions. (As before, $d$ denotes the dimension of the space and in our case $d=1,2$ or 3 . However, if $d=1$ the statement becomes trivial, so we shall only consider higher dimensions.)

Suppose our claim is false. Let $B(z, \delta)$ be a ball around $z$ of an arbitrarily small radius $\delta$. Then $B(z, \delta) \cap F$ is at most a $(d-1)$-dimensional subset of $\mathbf{R}^{d}$, so it has measure zero. This, however, contradicts Lebesgue's Theorem on points of density, which states that almost all points of any arbitrary linear set are density points of that set, i.e. for a.e. $z \in F$

$$
\lim _{\delta \rightarrow 0} \frac{\mu(F \cap B(z, \delta))}{\mu(B(z, \delta))}=1 .
$$

Thus our claim is established.

The above claim justifies the next assertion, namely that $\nabla \psi$ can be determined a.e. on $F$ by considering only sequences of points of $F$. Repeating the same argument one more time we find that $\sqrt{\alpha} \Delta \psi$ (or $\sqrt{-\alpha} \psi$ where $\alpha \psi^{2}<0$ ) is real a.e. on $F$. Then we see that in

$$
\sqrt{\alpha}\left(-\Delta+V_{\#}-E_{\#}\right) \psi=-i \sqrt{\alpha} \varepsilon_{\#} \psi
$$

the left side would have to be real and the right side imaginary, which means that $\psi=0$.

Equation (3.2) is consistent with the expectation that maximally resonant potentials act by confining a particle inside a barrier, i.e., that the potential lies predominantly near the periphery of $\Omega$, but in principle the set $Y$ at this point need have no special position within $\Omega$. The spherically symmetric analysis will bear out the expectation more fully. In one dimension $Y$ will in fact turn out to be (a.e. equivalent to) a finite union of closed intervals (Proposition IV.2).

Proposition III.3. With $\alpha$ as in the foregoing proof, $\operatorname{Im}\left(\alpha \psi^{2}\right) \geqslant 0$ on the set $Y$ of (3.2), and $\operatorname{Im}\left(\alpha \psi^{2}\right) \leqslant 0$ on the complement of $\bar{Y}$. Moreover, $\alpha \psi^{2}$ is real on the boundary of $Y$.

REMARK. It would thus be possible to modify the normalization of (1.3) and (2.11) so as to make $\operatorname{Im} \psi^{2}$ respectively $\geqslant 0$ and $\leqslant 0$.

PROof. For $Z \subset Y$, we may allow a perturbation of the form $V_{\#} \rightarrow V_{\#}+\kappa \chi_{Z}$ so long as $\kappa \leqslant 0$, so that the potential remains in $S$. As in the proof of Theorem III.2, we find that for a.e. $x \in Y, \operatorname{Im}\left(\alpha \psi^{2}\right) \geqslant 0$. Similarly, for $Z \subset \bar{Y}^{\prime}$ we may allow such perturbations so long as $\kappa \geqslant 0$, and the argument of the proof of Theorem III.2 shows that for a.e. $x \notin Y, \operatorname{Im}\left(\alpha \psi^{2}\right) \leqslant 0$. Therefore, by the continuity of $\psi_{\#}, \alpha \psi_{\#}^{2}$ is real on the boundary of $Y$. 
IV. The spherically symmetric case. Finally, we embark on the detailed description of the totally spherically symmetric case via a series of propositions and remarks. We will find that the wave-functions of maximal resonances not only suffer from confinement, but they also get kicked when they are down. We show below that, at least for large $L$ or $M$, maximally resonant potentials must contain a confining barrier stretching to $L$. We believe that there are locally maximally resonant potentials consisting of more than one barrier, although we do not firmly establish this fact. In particular, as can be seen from (4.1) and (4.3) below, the potential can and will switch on inside the outer barrier if the resonance wave-function has a sufficiently small modulus over a given region. This will happen if the resonance wave-function resembles an excited state of the associated problem with some selfadjoint boundary condition at $L$, which is ordinarily the case when the resonance width is small. The reason for this conjecture is provided, for example, by [1], where resonances are localized near, and asymptotically in one-to-one correspondence with, bound state energies of a related selfadjoint problem. The sharpest resonance of all seems to be generally associated with the ground-state eigenfunction, and its potential contains a confining barrier but no other pieces.

One of the tools for deriving more information about the set $Y$ if there is total spherical symmetry is the formula (2.11) relating any resonance width to the corresponding resonance function on $[0, L]$. It leads to the following:

Proposition IV.1. In the spherically symmetric case, the argument of any resonance eigenfunction is monotone increasing and twice differentiable. More exactly,

$$
d \arg (\psi) / d r=\varepsilon|\psi(r)|^{-2} \int_{0}^{r}|\psi(y)|^{2} d y>0
$$

Proof. First note that $\psi(r)$ never vanishes except at $r=0$, as otherwise it would be an eigenfunction of a selfadjoint problem, and $\varepsilon$ would have to be 0 . If $u=d(\arg \psi) / d r=d(\operatorname{Im} \ln \psi) / d r=\operatorname{Im}\left(\psi^{\prime} / \psi\right)$, then, after the usual Ricatti transformation, the Schrödinger equation becomes

$$
u^{\prime}=\varepsilon-\left(2 \operatorname{Re}\left(\psi^{\prime} / \psi\right)\right) u \text {. }
$$

Formula (2.11) fixes the limit of integration in the solution of this elementary equation, leading to (4.1).

Proposition IV.2. In the spherically symmetric case, the support $Y$ of $V_{\#}-W$ is a finite union of disjoint intervals, i.e., for some integer $n \geqslant 1$, there are points $0 \leqslant r_{1}<r_{2}<\cdots<r_{2 n} \leqslant L$ for which, if we let $B(j)=\left[r_{2 j-1}, r_{2 j}\right], \quad G(j)=$ $\left[r_{2 j}, r_{2 j+1}\right]$, then

$$
Y=\bigcup_{j=1}^{n} B(j) .
$$

In addition, the following estimates hold for the lengths of the intervals $B(j)$ and gaps $G(j)$ : For all $j$ except (i) $j=1$ when $r_{1}=0$, or (ii) $j=n$ when the associated interval or gap includes the value $L$,

$$
|B(j)|>\pi \min _{B(j)}\left|\psi_{\#}\right|^{2} / 2 K \text { and }|G(j)|>\pi \min _{G(j)}\left|\psi_{\#}\right|^{2} / 2 K
$$

where, as before, $K=\operatorname{Re} k$. 
Definition. We call the intervals $B(j)$ the barriers and the intervals $G(j)$ the gaps.

Proof. From Propositions III.3 and IV.1 it follows that in one dimension the potential switches on or off exactly at the places where the argument of $\psi_{\#}$ increases by $\pi / 2$ from the first point at which it switches on or off. Since $\psi_{\#}$ satisfies a regular Sturm-Liouville equation and vanishes at 0 , it is continuously differentiable with $\psi_{\#}^{\prime}(0) \neq 0$ (else it would vanish everywhere). It follows that the expression in (4.1) is bounded for all $r$, so there can only be a finite number of switchings. This establishes (4.2).

The estimates (4.3) follow from (4.1). The limiting phase at $r=0$ is undetermined, so the first switching point is likewise undetermined. Also, the potential is switched off by construction at $L$ regardless of phase. For the other switching points, however, (4.1) implies that

$$
\pi / 2=\varepsilon \int_{B(j) \text { or } G(j)} d r|\psi(r)|^{-2} \int_{0}^{r}|\psi(y)|^{2} d y .
$$

Now replace $r$ by $L$ and substitute from (2.11) to get

$$
\pi / 2<K \int_{B(j) \text { or } G(j)} d r|\psi(r)|^{-2},
$$

and, finally, estimate the remaining integral by the length of the interval times the maximum of the integrand.

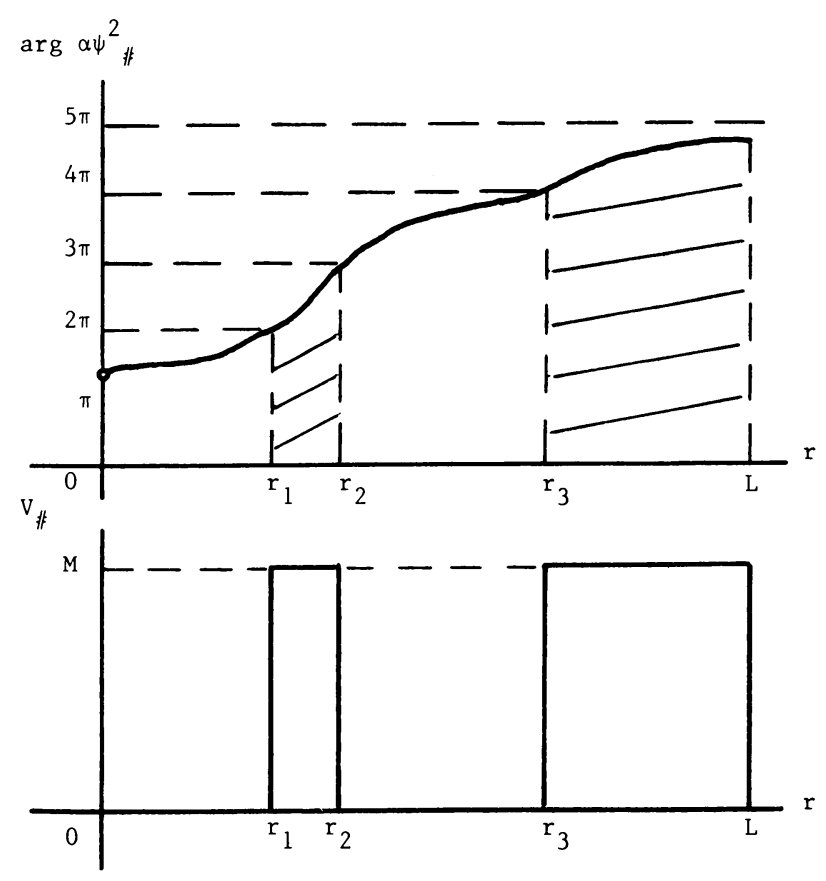

FIGURE 1. The relationship between the argument of the resonance function and the on and off intervals of the maximally resonant potential. The potential equals $M$ in the shaded intervals and 0 otherwise. 


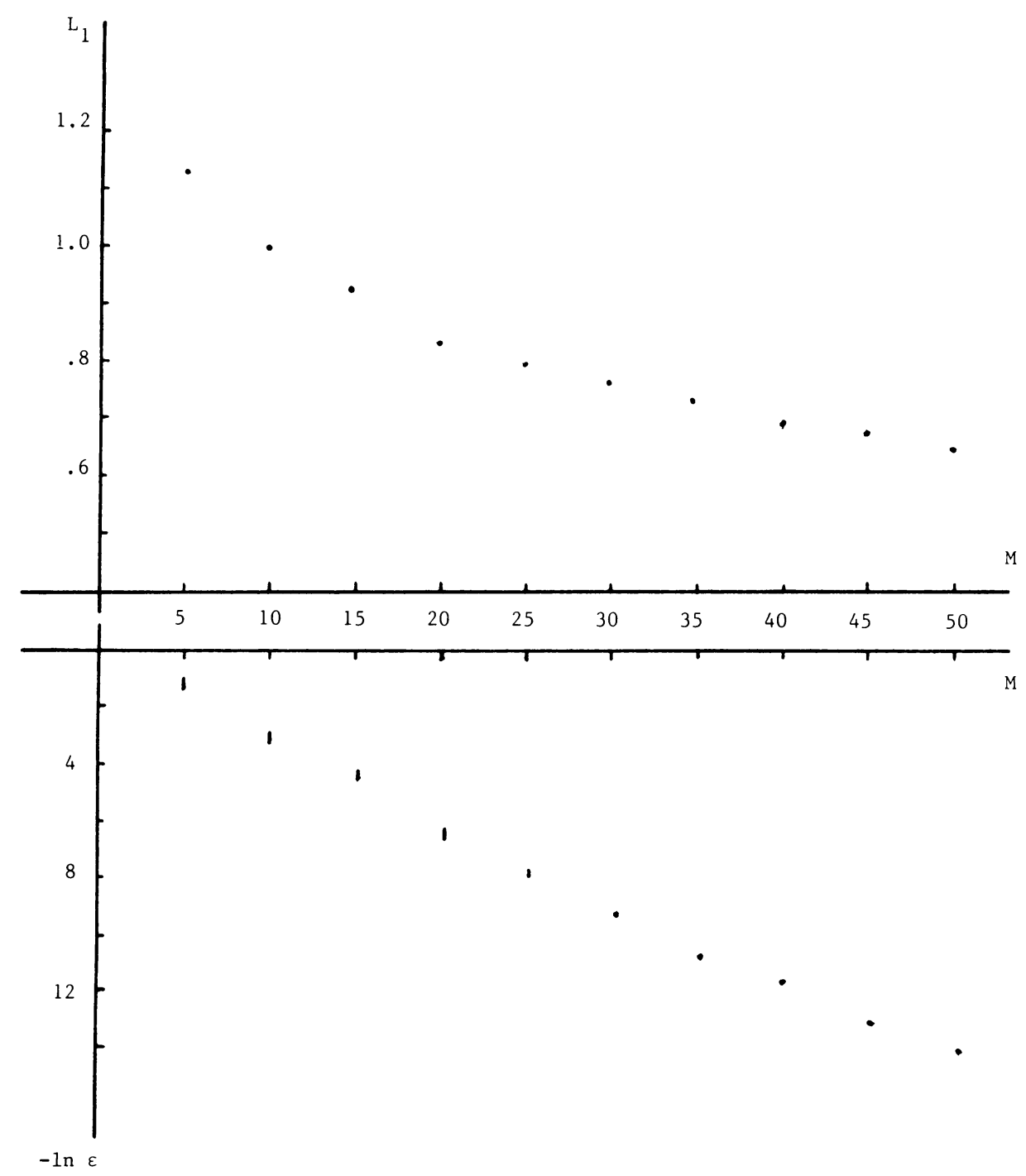

Figure 2. We fix $L=2$ and assume that $V=M \chi_{\left[I_{1}, 2\right]}$. Then we numerically evaluate $\varepsilon\left(L_{1}\right)$ and $d \varepsilon / d L_{1}$ for different values of $L_{1}$. Points on the first graph represent optimal values of $L_{1}$ for each fixed value of $M$ and points on the second graph represent corresponding values of $\ln \varepsilon$.

From now on we set $W=0$. Once $k^{2}$ is determined for a (locally) maximally resonant potential, there is a simple algorithm for determining the positions of the finite number of "on" and "off" intervals. Since $\psi_{\#}$ is respectively either a linear combination of exponential functions $\exp \left( \pm k^{\prime} r\right), k^{\prime}=\sqrt{M-k^{2}}$, or a combination of sinusoidal functions $\sin (k r)$ and $\cos (k r)$ and is continuously differentiable at the switch points, it is a matter of algebra to determine the argument at any given point. The argument steadily increases from the point $r=0$, and the potential switches on and off whenever it increases by $\pi / 2$. The limiting initial phase at $r=0$ is determined by the condition that the eigenfunction satisfies the resonance condition at $r=L$. 
Definition. A resonance will be called typical if $L \sqrt{M}>\pi / 2$ and its real part satisfies

$$
\pi^{2} / 4 L^{2}<E<0.9 M
$$

and

$$
\max \left(-\operatorname{Im}(k / \sqrt{M}), \operatorname{Im}\left(k^{\prime} / \sqrt{M}\right),-\operatorname{Im}\left(k / k^{\prime}\right), \operatorname{Im}\left(k^{\prime} / k\right)\right)<\exp \left(-L^{1 / 2} M^{1 / 4}\right),
$$

where $k^{\prime}=\left(M-k^{2}\right)^{1 / 2}$ (conventionally in the first quadrant).

It is not hard to see from Proposition II.2 that for large $L$ or $M$ maximally sharp resonances in this energy range have to be typical, and tunneling estimates indicate that resonances above this energy range are not extremely sharp (some bounds on widths will appear in [15]). In particular, the sharpest resonance of all is typical when $L$ or $M$ is sufficiently large. Our last claim states that typical maximally sharp resonances are due at least in part to barrier confinement:

Proposition IV.3. If a totally spherically symmetric resonance is typical and locally maximal, then $r_{2 n}$ (cf. Proposition IV.2) equals $L$.

Proof. Suppose not. Then the outermost barrier ends at a point $z<L$. There are then two possibilities: either (a) there is only one barrier stretching from 0 to $z$, or (b) the argument of $\psi_{\#}$ increases by $\pi / 2$ on the barrier $[y, z]$ with $y>0$. Possibility (a) is easily checked not to be typical (or maximally sharp), so (b) would have to prevail. But if $z$ is the outermost edge of the potential, then $\psi_{\#}$ satisfies an outgoing condition at $z$ of the form $\psi_{\#}^{\prime}(z) / \psi_{\#}(z)=i k$. We may modify (1.3) by a fixed multiplicative constant and assume that $\psi_{\#}(z)=1$, which means that on $[y, z]$, $\psi_{\#}(r)=\cosh \left(k^{\prime}(z-r)\right)-i\left(k / k^{\prime}\right) \sinh \left(k^{\prime}(z-r)\right)$. Hence $\cosh \left(k^{\prime}(z-y)\right)-$ $i\left(k / k^{\prime}\right) \sinh \left(k^{\prime}(z-y)\right)$ must be purely imaginary. Taking the real part and dividing by a real quantity, we find that

$$
0=1+\tanh \left(\operatorname{Re}\left(k^{\prime}\right)(z-y)\right) \operatorname{Im}\left(k / k^{\prime}\right)+\tan \left(\operatorname{Im}\left(k^{\prime}\right)(z-y)\right) \operatorname{Re}\left(k / k^{\prime}\right) .
$$

This is impossible if (4.5) holds, as can be seen by substitution and straightforward estimates.

We close with the result of a representative numerical study of the maximally resonant potentials in the totally spherically symmetric case. We fix $L=2$ and consider various barrier heights $M$. Tunneling estimates indicate that the maximal resonance for these values arises from a single barrier with $V=M \chi_{\left[L_{1}, 2\right]}$. The optimal values of $L_{1}$ and the corresponding $\varepsilon$ are depicted in Figure 2. The error bars are numerical estimates but are not rigorously established.

\section{REFERENCES}

1. M. S. Ashbaugh and E. M. Harrell II, Perturbation theory for shape resonances and large barrier potentials, Comm. Math. Phys. 83 (1982), 151-170.

2. _. Maximal and minimal eigenvalues and their associated nonlinear equations, preprint 1985.

3. E. Balslev, Local spectral deformation techniques for Schrödinger operators, Inst. Mittag-Leffler Report 14, 1982, Resonances, Resonance Functions, and Spectral Deformations, Zentrum für interdisziplinäre Forschung, preprint, 1984. 
4. Hans Cycon, Resonances defined by modified dilations, preprint, 1984.

5. M. S. P. Eastham and H. Kalf, Schrödinger-type operators with continuous spectra, Pitman, Boston, Mass., 1982.

6. S. Graffi and K. Yajima, Exterior complex scaling and the AC-stark effect in a Coulomb field, Comm. Math. Phys. 89 (1983), 277-301. ${ }^{3}$

7. E. M. Harrell II, General lower bounds for resonances in one dimension, Comm. Math. Phys. 86 (1982), 221-225.

8. __ Hamiltonian operators with maximal eigenvalues, J. Math. Phys. 25 (1984), 48-51.

9. P. Hartman, Ordinary differential equations, Wiley, New York, 1964.

10. W. Hunziker, private communication.

11. T. Kato, Perturbation theory for linear operators, Springer, New York, 1966.

12. M. Reed and B. Simon, Methods of modern mathematical physics, four volumes, Academic Press, New York, 1972-1979.

13. I. Sigal, Complex transformation method and resonances in one-hody quantum systems, Weizmann Institute, preprint, 1983.

14. B. Simon, The definition of molecular resonance curves by the method of exterior complex scaling, Phys. Lett. A71 (1979), 211-214.

15. R. Svirsky, Johns Hopkins dissertation, 1985.

16. E. C. Titchmarsh, Eigenfunction expansions associated with second order differential equations, Part II, Oxford Univ. Press, 1958.

School of Mathematics, Georgia Institute of Technology, Atlanta, Georgia 30332-0160 (Current address of E. M. Harrell II)

Department of Mathematics, The Johns Hopkins University, Baltimore, Maryland 21218

Current address (Roman Svirsky): Department of Mathematics, Tulane University, New Orleans, Louisiana 70118

\footnotetext{
${ }^{3} \mathrm{We}$ are informed by the authors that there are some technical lacunae in this paper. They do not, however, affect the exteriorly complex-scaled resolvent as defined in their equation (2.15).
} 